\title{
Use of Oritavancin in Acute Bacterial Skin and Skin Structure Infections Patients Receiving Intravenous Antibiotics: A US Hospital Budget Impact Analysis
}

\author{
Ivar S. Jensen ${ }^{1}$ Thomas P. Lodise ${ }^{2}$ - Weihong Fan $^{3}$ - Chining $\mathrm{Wu}^{1}$ • \\ Philip L. Cyr ${ }^{1} \cdot$ David P Nicolau $^{4} \cdot$ Scott DuFour $^{5} \cdot$ Katherine A. Sulham $^{3}$
}

Published online: 21 December 2015

(c) The Author(s) 2015. This article is published with open access at Springerlink.com

\begin{abstract}
Background and Objective Nearly $10 \%$ of all US hospital admissions are attributed to acute bacterial skin and skin structure infections (ABSSSIs). While most antibacterials used to treat these infections require multi-day and multidose regimens, a single-dose treatment is now available. The objective of this analysis is to estimate the annual budget impact of using single-dose oritavancin in patients with moderate to severe ABSSSIs receiving intravenous methicillin-resistant Staphylococcus aureus (MRSA)-active antibacterials from a US hospital perspective.

Methods A decision-analytic model based on current clinical practice was developed to estimate the economic impact of oritavancin. Utilization of antibacterials and rates of hospital admission were derived from the Premier Research Database. Demographic and clinical data were informed by the published literature and 2014 wholesale drug acquisition costs were used. Other costs were based on the published literature and Medicare National Limitation amounts. All
\end{abstract}

Electronic supplementary material The online version of this article (doi:10.1007/s40261-015-0365-8) contains supplementary material, which is available to authorized users.

Katherine A. Sulham

kate.sulham@themedco.com

ICON Health Economics, Cambridge, MA, USA

2 Albany College of Pharmacy and Health Sciences, Albany, NY, USA

3 The Medicines Company, 8 Sylvan Way, Parsippany, NJ, USA

4 Center for Anti-Infective Research and Development, Hartford Hospital, Hartford, CT, USA

5 Pharmaceutical Services and Ambulatory Infusion Center, Beaumont Health System, Troy, MI, USA costs were inflated to 2014 US dollars. Two base-case scenarios were considered: one for hospitals with ambulatory services and one for hospitals without ambulatory services. Results For a US hospital with ambulatory services with 1000 ABSSSI patients receiving intravenous MRSA antibiotics annually, use of oritavancin in $26 \%$ of patients is estimated to reduce the total annual budget by $12.9 \%$ (US\$1.23 million), or approximately US $\$ 1234.67$ per patient. Total inpatient costs will be reduced by $22.3 \%$ (US $\$ 1.40$ million) and outpatient costs will increase slightly by $1.7 \%$ (US\$55,310). Pharmaceutical cost increases are offset by savings in the inpatient setting from fewer hospital admissions. Hospitals without ambulatory services are estimated to receive overall cost savings of $9.3 \%$ (US $\$ 0.63$ million).

Conclusion Use of single-dose oritavancin in select ABSSSI patients with suspected or confirmed MRSA involvement is estimated to save US hospitals approximately $9.3-12.9 \%$ per year by reducing hospital admissions and lowering drug administration burden.

\section{Key Points for Decisions Makers}

Use of oritavancin in moderate to severe acute bacterial skin and skin structure infections (ABSSSIs) patients is expected to save hospitals substantial costs by reducing hospital admissions and decreasing costs associated with outpatient therapy.

Use of oritavancin may facilitate outpatient treatment of ABSSSI, thereby reducing the cost and resource use associated with hospital admissions.

Due to its single-dose administration, use of oritavancin may eliminate the need for daily intravenous drug administration and associated costs. 


\section{Introduction}

Management of patients with serious skin infections is a major burden on the US healthcare system. The Centers for Disease Control have reported that there are over 3.4 million emergency department (ED) visits annually for cellulitis and abscesses, making skin infections the seventh leading primary diagnosis seen in the ED [1]. Nearly $10 \%$ of all US hospital admissions are attributed to acute bacterial skin and skin structure infections (ABSSSIs), and total US hospital costs for treating ABSSSI patients in 2010 alone were estimated to be more than US\$6 billion $[2,3]$. ABSSSIs are predominantly caused by Gram-positive pathogens, with Staphylococcus aureus the most prevalent organism [5]. In recent decades, methicillin-resistant $S$. aureus (MRSA) strains have become increasingly common and, if un- or under-treated, may become lifethreatening. The overall national prevalence of MRSA has been reported to be $59 \%$; however, the rate varies by hospital and geography and can reach up to $74 \%$ or more of cases [6]. The high volume of ED visits, hospital admissions, and high costs require hospitals and healthcare systems to ensure clinically effective and cost-effective management of ABSSSI. Given the high prevalence of MRSA, any agent used in an effort to reduce the healthcare burden associated with treatment of ABSSSI must have reliable activity and demonstrated efficacy against this highly virulent pathogen [6].

Currently, the Infectious Diseases Society of America (IDSA) guidelines and associated drug package inserts recommend a treatment course of a minimum of 5-14 days of antibacterials such as vancomycin, daptomycin, ceftaroline, or intravenous/oral linezolid whether or not MRSA has been confirmed [7-12]. The multi-dose and multi-day treatment regimens required of most antibacterial drugs leads to multi-day hospital stays and often repeated visits to an infusion center to complete the course of therapy following discharge.

Oritavancin (Orbactiv®), The Medicines Company, Parsippany, NJ, USA) is a recently approved intravenous semi-synthetic lipoglycopeptide antibacterial indicated for the treatment of adult patients with ABSSSI caused by certain Gram-positive pathogens including MRSA [13]. As the first and only US FDA-approved single-dose treatment for ABSSSI, it has been hypothesized that oritavancin may reduce costs for ABSSSI treatment through the avoidance of hospitalization or shortened length of stay (LOS) made possible by its once-only dosing, particularly in stable patients with moderate to severe ABSSSIs $[4,7]$. To explore this hypothesis, a budget impact model was developed from the hospital perspective to estimate the economic impact of using once-only oritavancin in moderate to severe ABSSSI patients. The objective of the analysis was to understand the cost impact of shifting an increased proportion of patients to care in the ambulatory setting, as compared to the current standard of care, based on nationally representative practice patterns.

\section{Methods}

A decision-analytic model based on current clinical practice was developed to simulate treatment of ABSSSI patients receiving empiric treatment with MRSA-active antibacterials (Fig. 1). The perspective of the model is from a US hospital, and costs considered include the index treatment episode and 30-day ABSSSI-related re-hospitalization. The decision tree was developed based on literature review and expert clinical opinion (SD, DPN, and TPL).

The model was used to estimate the annual budget impact of using oritavancin for a hypothetical US hospital with 1000 patients per year receiving intravenous MRSAactive antibacterials for ABSSSI. Since incidence of the disease varies by hospital, 1000 patients was selected as the population in order to facilitate comparison with individual hospitals. The base case reflects a nationally representative empiric treatment mix of $92 \%$ vancomycin, $2 \%$ linezolid intravenous, and $6 \%$ daptomycin (Table 1). In the scenario case, oritavancin was assumed to be used in $26 \%$ of patients, representing those patients with moderate to severe ABSSSI who can safely be managed in an outpatient environment, as derived from an analysis of the Premier Research Database [14]. In this analysis, approximately $26 \%$ of admitted patients with ABSSSIs were Eron classification 2 and 3, a group of patients with moderate to severe ABSSSI with the potential to receive treatment in the outpatient setting due to lack of other reasons for hospital admission. Oritavancin usage in this scenario was assumed to displace vancomycin while all other antibacterial use was unchanged, resulting in a scenario treatment mix of $66 \%$ vancomycin, $26 \%$ oritavancin, $2 \%$ linezolid intravenous, and $6 \%$ daptomycin. We elected to shift $26 \%$ of patients from vancomycin to oritavancin use rather than the other antibacterials for two reasons. First, vancomycin was the clinical comparator in the SOLO trials of oritavancin $[15,16]$. In these trials, oritavancin was shown to be non-inferior to vancomycin for ABSSSI. Therefore, oritavancin will likely replace vancomycin in clinical use due to the available comparator data. Second, vancomycin was the least expensive of the drugs included in this analysis. We thus felt that comparing a new more costly alternative to the least expensive established treatment was the most economically conservative assumption. If oritavancin is found to be cost saving 


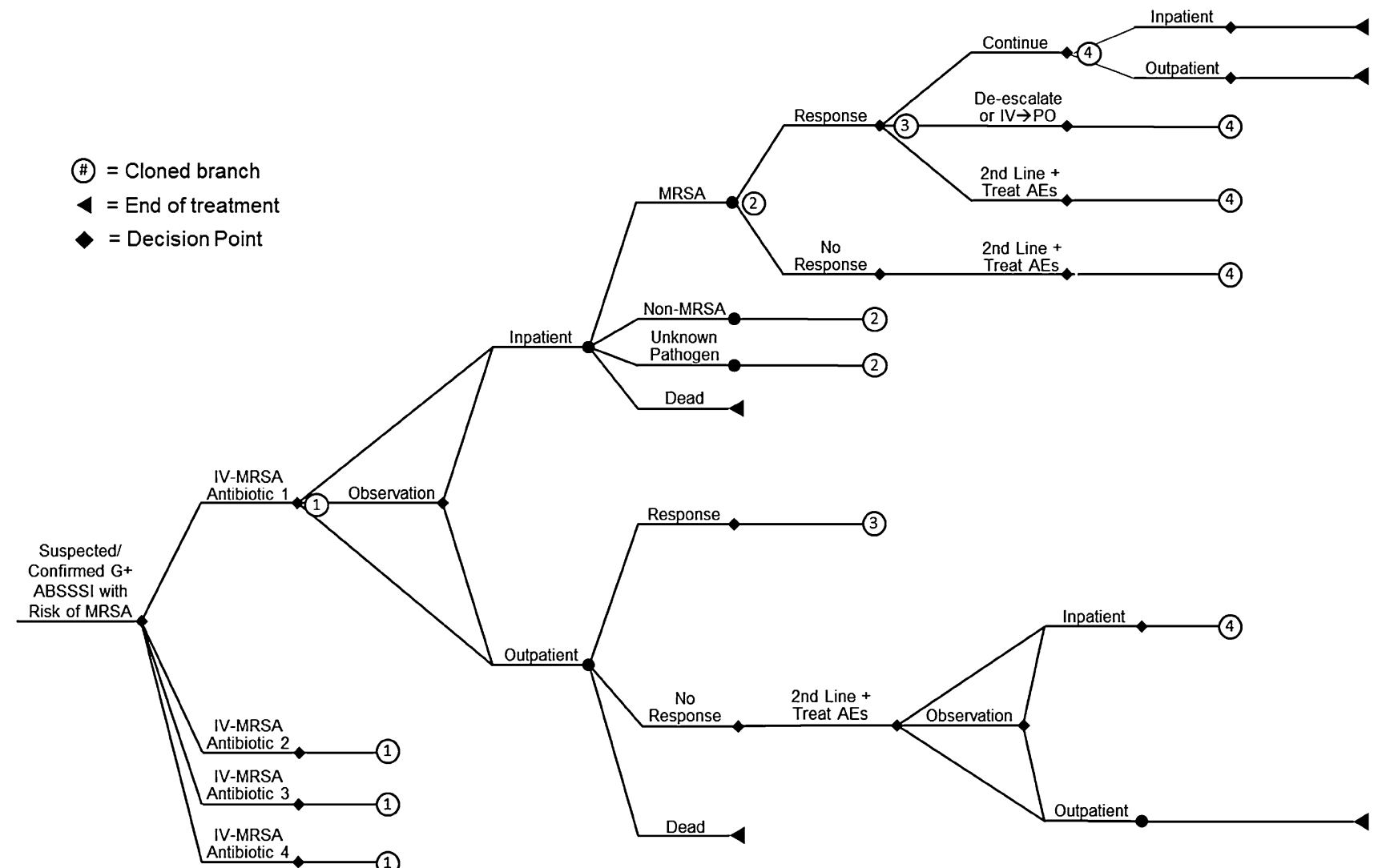

Fig. 1 Decision-analytic framework for acute bacterial skin and skin structure infection patient management. ABSSSI acute bacterial skin and skin structure infection, $A E$ adverse event, $G+$ Gram-positive

relative to vancomycin, it will also be cost saving relative to linezolid and daptomycin. Based on expert clinical opinion, oritavancin patients were assumed to be treated in the following settings and frequencies: hospital inpatient (5\%), observation $(80 \%)$, and outpatient $(15 \%)$. It was assumed that $100 \%$ of observation patients were discharged to outpatient care.

In order to capture the experience of hospitals with and without financial risk for outpatient care, two base-case scenarios were assessed:

1. Hospital with ambulatory services. This represents a hospital at full financial risk for the patients' entire course of care (e.g., patients return to a hospital-owned setting for ambulatory treatment, such as a hospitalowned outpatient parenteral antimicrobial therapy [OPAT] infusion center). For these hospitals, outpatient costs could accrue through hospital outpatient departments such as the ED and observation unit as well as in the ambulatory care setting, such as an OPAT infusion center.

2. Hospital without ambulatory services. This represents a hospital that bears $0 \%$ of costs incurred outside the pathogens, IV intravenous, MRSA methicillin-resistant Staphylococcus aureus, $P O$ oral

inpatient setting (e.g., patients are discharged to an independently owned ambulatory infusion setting, such as a physician-owned OPAT infusion center or a home health infusion agency). Patients in hospitals without ambulatory services could still incur outpatient costs in the ED or when they are under observation; however, the costs and resource use associated with continued ambulatory services provided outside of the hospital would not accrue to the hospital budget.

\subsection{Model Structure}

As illustrated in Fig. 1, ABSSSI patients entering the ED are treated with empiric MRSA-active therapy and may be cultured for pathogen confirmation and susceptibility testing. After receiving one dose of empiric therapy in the ED, patients may be treated in one of three settings: hospital inpatient, observation, or outpatient. The model includes the following outcomes for responders: (1) continue empiric therapy; (2) de-escalate therapy (including either switching to non-MRSA active therapy, a less frequent dosing regimen, or oral therapy); or (3) switch therapy due 
Table 1 Key clinical inputs (\%)

\begin{tabular}{|c|c|c|c|c|c|}
\hline Utilization parameters & Oritavancin & Vancomycin & Linezolid & Daptomycin & Source \\
\hline Empiric treatment setting & & & & & [19], expert opinion \\
\hline Inpatient & 5.0 & 55.9 & 77.0 & 19.0 & \\
\hline Observation & 80.0 & 66.0 & 7.0 & 1.0 & \\
\hline Outpatient & 15.0 & 33.5 & 16.0 & 80.0 & \\
\hline Final treatment setting & & & & & [19], expert opinion \\
\hline Inpatient & 5.0 & 22.6 & 28.0 & 35.0 & \\
\hline Outpatient & 95.0 & 77.4 & 72.0 & 65.0 & \\
\hline Empiric therapy utilization rate (base case) & 0.0 & 92.0 & 2.0 & 6.0 & {$[19]$} \\
\hline Empiric therapy utilization rate (scenario) & 25.8 & 66.3 & 2.0 & 6.0 & Assumption \\
\hline Response rate & & & & & {$[17]$} \\
\hline MRSA & 74.5 & 76.0 & 83.0 & 68.4 & \\
\hline Non-MRSA & 85.0 & 89.0 & 90.0 & 94.3 & \\
\hline Unknown pathogen & 82.9 & 82.2 & 87.4 & 90.2 & \\
\hline Mortality & & & & & {$[15,16,19,33]$} \\
\hline MRSA & 0.4 & 0.9 & 0.4 & 0.4 & \\
\hline Non-MRSA & 0.4 & 0.9 & 0.4 & 0.4 & \\
\hline Unknown pathogen & 0.2 & 0.3 & 0.4 & 0.4 & \\
\hline AE discontinuation & 0.0 & 6.0 & 3.0 & 2.8 & {$[10,36,37]$} \\
\hline 30-day ABSSSI-related readmission rate & 1.6 & 3.0 & 4.0 & 4.2 & {$[34,35,38,39]$} \\
\hline $\mathrm{AE}$ rates & & & & & {$[10,12,13]$} \\
\hline Elevated CPK & 0.6 & 0.0 & 0.0 & 2.8 & \\
\hline Myelosuppression & 0.0 & 0.0 & 3.5 & 0.0 & \\
\hline Acute kidney injury & 0.0 & 1.0 & 0.0 & 0.0 & \\
\hline Phlebitis & 0.0 & 9.2 & 4.5 & 4.3 & \\
\hline Rash/pruritus & 3.0 & 6.0 & 2.0 & 4.5 & \\
\hline Nausea/vomiting & 7.3 & 7.6 & 5.0 & 5.2 & \\
\hline Diarrhea & 3.7 & 3.3 & 2.2 & 0.0 & \\
\hline Constipation & 3.4 & 3.9 & 0.0 & 2.4 & \\
\hline
\end{tabular}

$A B S S S I$ acute bacterial skin and skin structure infections, $A E$ adverse event, $C P K$ creatine phosphokinase, MRSA methicillin-resistant Staphylococcus aureus

to intolerance. Responders who de-escalate therapy are assumed to continue to respond. Non-responders and patients who discontinue due to intolerance require switching to a second-line intravenous antibacterial, and these patients are assumed to be cured following secondline therapy. Patients who are placed under observation may progress to treatment as inpatients or to discharge for continued ambulatory treatment. Following clinical practice, admitted patients may complete their full course of therapy in the hospital or they may be discharged to complete their treatment as outpatients. For patients discharged to outpatient from the ED or observational unit, responders may continue therapy, de-escalate, or switch intravenous antibiotic therapy due to adverse events (AEs). Non-responding outpatients will require a switch of therapy, and may then become hospitalized or kept as outpatients, with or without a period of observation. ABSSSI patients are followed up through the treatment paradigm until 30 days after completion of therapy; 30-day readmission rates specific to the final therapy were applied for all patient pathways. Table 1 lists the key clinical inputs used in the model.

\subsection{Clinical Inputs}

Clinical inputs were obtained from a systematic literature review and network meta-analysis (NMA) [17]. In brief, the NMA utilized Bayesian indirect treatment effects to determine the comparative efficacy point estimates for MRSA infections, non-MRSA infections, and unknown infections for multiple antibacterials including vancomycin, linezolid, daptomycin, oritavancin, and others. 
Table 2 Key healthcare resources

\begin{tabular}{|c|c|c|}
\hline Parameters & Value & Source \\
\hline \multicolumn{2}{|l|}{ Average days of treatment } & \multirow[t]{5}{*}[18,40]{, expert opinion } \\
\hline Oritavancin (IV) & 1 & \\
\hline Vancomycin (IV) & 10.2 & \\
\hline Linezolid (IV) & 13.7 & \\
\hline Daptomycin (IV) & 11.1 & \\
\hline \multicolumn{2}{|l|}{ Laboratory tests (per week) } & \multirow[t]{6}{*}[17,40]{, expert opinion } \\
\hline Chem7 & $1 \times$ vancomycin; $1 \times$ daptomycin; $1 \times$ linezolid & \\
\hline CBC levels & $1 \times$ linezolid & \\
\hline CPK levels & $1 \times$ daptomycin & \\
\hline Drug trough concentrations & $2 \times$ vancomycin & \\
\hline Hepatic panel & $1 \times$ daptomycin & \\
\hline \multicolumn{3}{|c|}{ Length of stay for hospitalized patients discharged to outpatient treatment (days) } \\
\hline Oritavancin (IV) & 2.5 & Assumption \\
\hline Vancomycin (IV) & 4.1 & {$[18]$} \\
\hline Linezolid (IV) & 4.42 & {$[18]$} \\
\hline Daptomycin (IV) & 4.5 & {$[18]$} \\
\hline Average time in observation (h) & 17.4 & {$[20,21]$} \\
\hline
\end{tabular}

$C B C$ complete blood count, $C P K$ creatine phosphokinase, $I V$ intravenous

Discontinuation, relapse, and AEs were not reported separately by pathogen type, and thus a single estimate informed by the literature search was used for each drug across all pathogen types (Table 1).

\subsection{Healthcare Resource Inputs}

Data derived from the Premier Research Database were used as the basis for determining the base-case first- and second-line treatment mix, days of treatment (DOT), LOS, and treatment setting for ABSSSI patients (Tables 1, 2) [18]. The Premier Research Database is geographically representative of the US and includes billing records for approximately one out of every five hospital discharges nationally [19]. Patients were included for analysis if they had a primary diagnosis relating to one of the following International Classification of Diseases, 9th revision, Clinical Modification (ICD-9-CM) codes: 035 erysipelas; 681.x and 682.x (cellulitis/abscess); 686.8 and 686.9 (other specified/unspecified local infections of skin and subcutaneous tissue); 958.3 (post-traumatic wound infection); 998.5x (post-operative infections); and an intravenous MRSA-active antibacterial prescription. Outcomes assessed include practice patterns for first- and secondline treatment and, by therapy, the proportion of patients treated as inpatients versus outpatients, total DOT, and total LOS. As average LOS in the real world is not yet known, an assumption of 2.5 days was used for oritavancin. Other intravenous antibacterial drugs had a ratio of inpatient days to days of therapy that ranged from 0.3 to 0.4. If applied to oritavancin, it would result in an unreasonable assumption of a less than 1-day stay. Conservatively, the assumption of 2.5 days of inpatient stay was inputted for oritavancin.

Time spent in observation, required laboratory monitoring and testing, drug administration frequency, and use of peripherally inserted central catheter (PICC) lines were also considered in the model. Published sources were used to estimate an average of $17 \mathrm{~h}$ for observation stay; this value was assumed to be equal across all treatments given lack of available data to suggest otherwise [20,21]. PICC lines were assumed to be used in all patients receiving multi-day infusions. Antibacterials were assumed to be administered according to the prescribing information, and frequencies of therapy-specific laboratory testing/monitoring were similarly inferred. Baseline laboratory testing was not included, as it was assumed to be equal for all patients. Table 2 lists the key health resource inputs included in the model.

\subsection{Economic Inputs}

Economic inputs utilized for the model base-case scenario are listed in Table 3. Published sources were used for PICC line costs for patients requiring an indwelling catheter [22]. Given the lack of available US-based microcosting data, Medicare National Limitation amounts were used as proxies for the cost for observation care, laboratory and drug administration costs, and hospital outpatient services. 
Table 3 Key economic inputs

\begin{tabular}{|c|c|c|c|}
\hline \multicolumn{2}{|l|}{ Parameters } & Value (US\$) & Source \\
\hline \multicolumn{4}{|l|}{ Drug acquisition costs } \\
\hline \multicolumn{2}{|l|}{ Vancomycin (IV) } & 18.66 & {$[24]$} \\
\hline \multicolumn{2}{|l|}{ Linezolid (IV) } & 263.38 & \\
\hline \multicolumn{2}{|l|}{ Daptomycin (IV) } & 354.72 & \\
\hline \multicolumn{2}{|l|}{ Oritavancin (IV) } & 2900.00 & \\
\hline \multicolumn{2}{|l|}{ Linezolid (PO) } & 271.15 & \\
\hline \multicolumn{4}{|l|}{ Laboratory costs } \\
\hline \multicolumn{2}{|l|}{ Chem7 } & 11.54 & [28] \\
\hline \multicolumn{2}{|l|}{ CBC levels } & 10.61 & \\
\hline \multicolumn{2}{|l|}{ CPK levels } & 8.88 & \\
\hline \multicolumn{2}{|c|}{ Drug trough concentrations } & 18.49 & \\
\hline \multicolumn{2}{|l|}{ Hepatic panel } & 11.14 & \\
\hline \multicolumn{4}{|c|}{ Drug administration costs } \\
\hline \multicolumn{2}{|c|}{ Peripherally inserted central catheter } & 412.15 & [22] \\
\hline \multicolumn{2}{|c|}{ IV infusion (initial hour) } & 172.18 & [28] \\
\hline \multicolumn{2}{|c|}{ IV infusion (each subsequent hour) } & 29.50 & \\
\hline \multicolumn{2}{|c|}{ IV bolus push (2-min bolus) } & 105.90 & \\
\hline \multicolumn{4}{|c|}{ Treatment setting cost per day } \\
\hline \multicolumn{2}{|l|}{ Observation cost } & 742.98 & [28] \\
\hline \multicolumn{2}{|l|}{ Inpatient cost } & 1231.81 & [3] \\
\hline Parameters & Fixed costs (US\$) & Daily cost (US\$) & Source \\
\hline \multicolumn{4}{|l|}{$\mathrm{AE}$ costs } \\
\hline Hypokalemia & 14.14 & 1.75 & \multirow[t]{9}{*}[22,24,28]{, expert opinion } \\
\hline Elevated CPK & NA & NA & \\
\hline Myelosuppression & 26.21 & NA & \\
\hline Acute kidney injury & $10,084.91$ & NA & \\
\hline Phlebitis & 416.16 & NA & \\
\hline Rash/pruritus & NA & 5.90 & \\
\hline Nausea/vomiting & NA & 34.80 & \\
\hline Diarrhea & 260.73 & NA & \\
\hline Constipation & NA & 1.91 & \\
\hline
\end{tabular}

$A E$ adverse event, $C B C$ complete blood counts, $C P K$ creatine phosphokinase, $I V$ intravenous, $P O$ orally

AE costs were based on the drug and laboratory costs of treating the condition. The Healthcare Cost and Utilization Project's (HCUP) 2011 National Inpatient Sample (NIS) dataset, the largest publicly available all-payer hospital inpatient care database in the US, was used to determine the cost of nephrotoxicity (IDC-9-CM 584.8 [acute kidney injury]). Costs associated with phlebitis were informed from the literature [23]. Pharmaceutical acquisition costs were based on the published wholesale acquisition cost (WAC), as the average sale price is inconsistently reported [24]. All costs were inflated to 2014 values using the medical consumer price index as reported by the Bureau of Labor Statistics [25].

\subsection{Sensitivity Analysis}

To assess the impact of uncertainty with model inputs on the results, a univariate sensitivity analysis was conducted where parameters were varied by $\pm 20 \%$ while keeping the remaining inputs constant. The range of $\pm 20 \%$ was selected as it would keep the inputs within plausible ranges as seen in the literature and as advised by clinical experts. For some parameters (e.g., response rate and others) where the $20 \%$ variation could not be feasibly applied, $100 \%$ was used for the upper range or $0 \%$ (e.g., mortality and others) as the lower range. Sensitivity analyses were conducted using Oracle Crystal Ball Release 11.1.2.4 for 


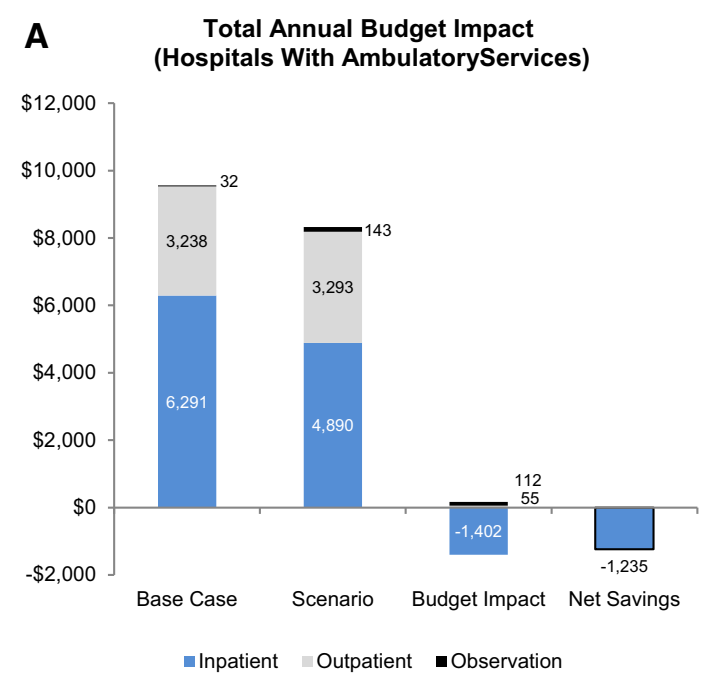

Fig. 2 Budget impact analysis for a US hospital with 1000 acute bacterial skin and skin structure infection patients eligible for intravenous methicillin-resistant Staphylococcus aureus-active antibacterials: a total annual costs and budget impact by setting and

Microsoft Office (Oracle Corporation, Redwood Shores, CA, USA).

\section{Results}

\subsection{Budget Impact Analysis}

Figure 2 illustrates the total estimated annual hospital costs for hospitals with (Fig. 2a) and without (Fig. 2b) ambulatory services.

\subsubsection{Hospital with Ambulatory Services}

For a hospital with ambulatory services, the annual budget is expected to decrease from US\$9.6 million in the base case to US\$8.3 million in the scenario, resulting in an overall net savings of $13 \%$, or US $\$ 1.3$ million (US $\$ 1235 /$ patient). The greatest savings came from inpatient hospitalization, where costs were reduced by $22 \%$ from US\$6.3 million to US\$4.9 million (Fig. 2a). Hospitalization was the greatest total annual cost category driver of cost reduction, followed by drug administration costs. Hospitalization and drug administration costs were reduced by $76 \%$ (US $\$ 1.1$ million) and $23 \%$ (US\$0.3 million), respectively (Fig. 3a). Total pharmaceutical costs increased $61 \%$ from US\$1 million in the base case to US\$1.6 million in the scenario due to the higher drug acquisition costs associated with oritavancin (Fig. 3a).

Although more patients in the scenario were treated entirely in an outpatient setting (47\% in the base case vs.

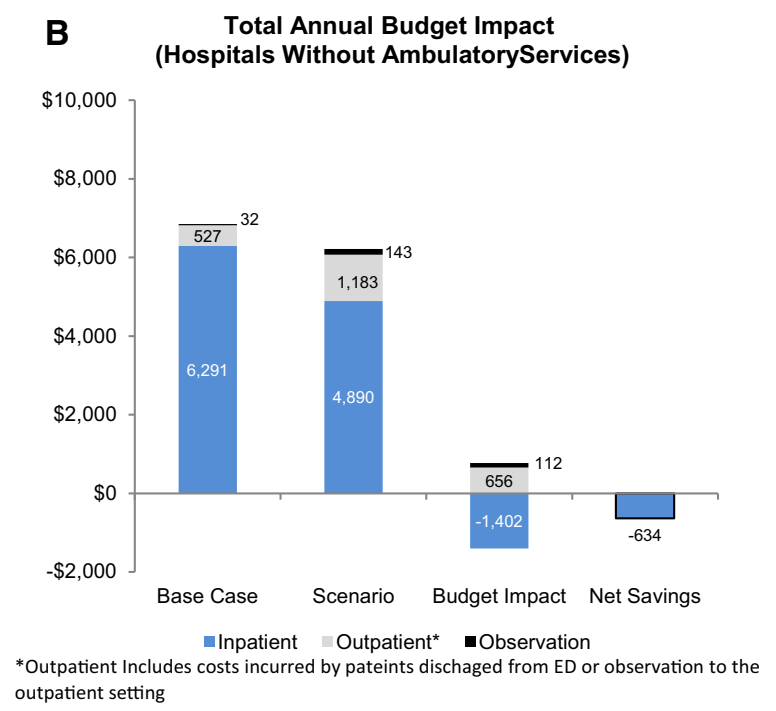

net savings for hospitals with ambulatory services; and $\mathbf{b}$ total annual costs and budget impact by setting and net savings for hospitals without ambulatory services. $E D$ emergency department

$68 \%$ in the scenario), total outpatient costs increased by only $2 \%$ (US $\$ 3.2$ million to US\$3.3 million). Total patient days were reduced for both inpatient and outpatient stays (inpatient: from 3629 to 2764 days; outpatient: from 6377 to 5087 days). Observation days increased from 43 days in the base case to 193 days in the scenario (data not shown), which led to a slight increase in annual observation unit costs of US\$111,780 (Fig. 3a). The number of laboratory tests was reduced by 4165 tests $(29 \%)$ in the scenario because oritavancin patients do not require monitoring. Other events such as readmissions were slightly improved with greater use of oritavancin (data not shown), largely through avoidance of any admission due to its once-only dosing

\subsubsection{Hospital Without Ambulatory Services}

A hospital without ambulatory services is not financially at risk for continued ambulatory treatment outside the hospital. For these hospitals, the total annual cost is also expected to decrease. The budget was reduced from US\$6.9 million in the base case to US\$6.2 million in the scenario, resulting in an overall net cost savings of $9 \%$, or US\$0.6 million (US\$634/patient). The inpatient hospitalization costs and savings mirror those of hospitals with ambulatory services, as given in Sect. 3.1.1, with inpatient costs reduced by $22 \%$ overall. Outpatient costs, including costs associated with activities in the ED and observation (but excluding continued ambulatory treatment costs), increased by US $\$ 0.7$ million (125\%), from US\$0.5 million in the base case to US\$1.2 million in the scenario 


\section{A \\ Total Annual Cost by Cost Category (Hospitals with Ambulatory Services)}

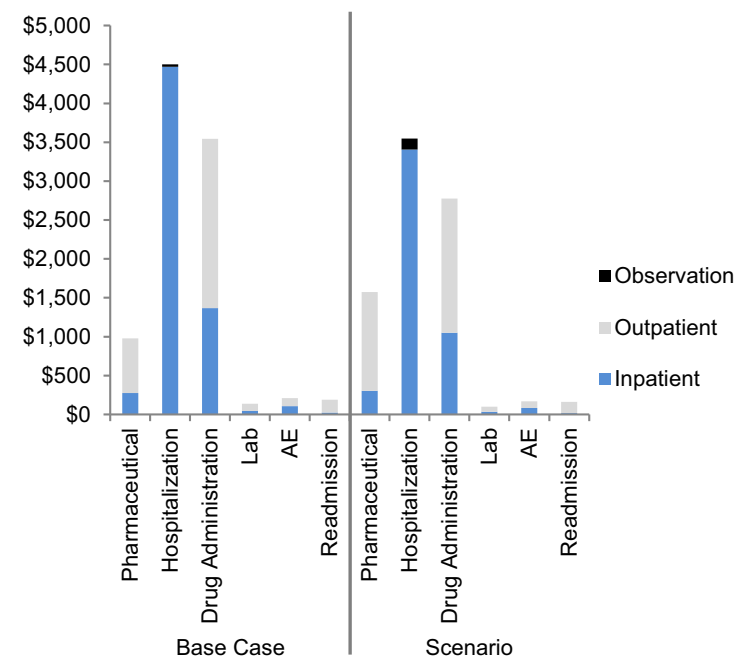

Fig. 3 Total annual cost by cost category for a US hospital with 1000 acute bacterial skin and skin structure infection patients receiving intravenous MRSA-active antibacterials: a total annual cost by cost category for hospitals with ambulatory services; and b total annual cost by cost category for hospitals without ambulatory services. For the base case and scenario, pharmaceutical represents the drug acquisition costs only for all antibacterials included in the model; hospitalization is the cost of inpatient stay excluding costs associated with pharmaceuticals, drug administration, laboratory tests, and

(Fig. 2b). The majority of this increase is due to the increased drug acquisition costs associated with oritavancin (US\$0.2 million in the base case to US\$0.7 million in the scenario) (Fig. 3b). Total patient days associated with inpatient and observation care were as outlined above (decrease of 865 inpatient days and increase of 150 observation days).

\subsection{Sensitivity Analysis}

Figure 4 and Table 4 illustrate the top 15 variables with the highest impact on the model results. Results are shown for hospitals with ambulatory services, though similar results were seen for hospitals without ambulatory services (data not shown). The model was most sensitive to the clinical response rates of antibacterials used in the largest proportions of patients in this analysis: oritavancin and vancomycin. As use of oritavancin was generally cost saving due to its once-only dosing and the shift of patients to the outpatient setting, the model was also sensitive to the proportion of patients receiving oritavancin, hospital cost per diem, and the unit cost (WAC) of oritavancin. Together, these five variables accounted for approximately $95 \%$ of variation in model outputs, with results ranging from US\$591,849 to US\$1,861,825 in cost savings with use of

\section{B Total Annual Cost by Cost Category}

(Hospitals without Ambulatory Services)

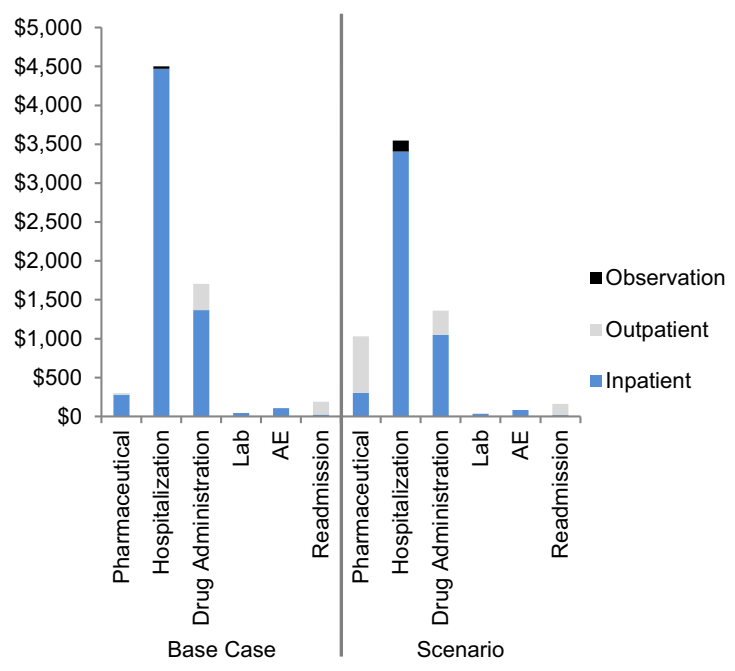

monitoring associated with MRSA-active antibacterials included in the model, adverse events, and readmissions; drug administration is the costs associated with drug administration, excluding pharmaceutical acquisition costs; $l a b$ is the costs of laboratory tests and monitoring associated with MRSA-active antibacterials included in the model; $A E$ is the cost associated with the adverse events listed in Table 1; and readmission represents the costs associated with 30-day re-hospitalization. $A E$ adverse event, MRSA methicillin-resistant Staphylococcus aureus

oritavancin in the base-case scenario. All other inputs have a relatively small impact on the model results; inputs not included in Fig. 4 had an impact of less than $1.5 \%$ on the total costs.

\section{Discussion}

This budget impact analysis was developed to assist hospital decision makers in evaluating the economic impact of using oritavancin in ABSSSI patients receiving empiric treatment with intravenous MRSA-active antibacterials. On average, a US hospital with ambulatory services and an annual caseload of 1000 ABSSSI patients receiving intravenous MRSA-active antibacterials could expect to save approximately $13 \%$ per year by using oritavancin in patients with moderate to severe ABSSSI $(26 \%$ of ABSSSI patients [14]) in a predominantly outpatient setting, equating to a per patient cost savings of US\$1235. Hospitals without ambulatory services could see similar, though slightly lower, cost savings with an expected net savings of $9 \%$ (US\$634 per patient).

While oritavancin has a comparable response rate to other antibacterial drugs, its once-only dosing regimen allows the majority of responders to avoid hospitalization 
Fig. 4 Univariate analysis: impact of a $\pm 20 \%$ change on total estimated costs for hospitals with ambulatory services. Only the top 15 most impactful variables on the total costs are included in figure. $O R I$ oritavancin, $V A N$ vancomycin, INPAT inpatient, DAP daptomycin, MRSA methicillinresistant Staphylococcus aureus, LIN linezolid, $O B S$ observation

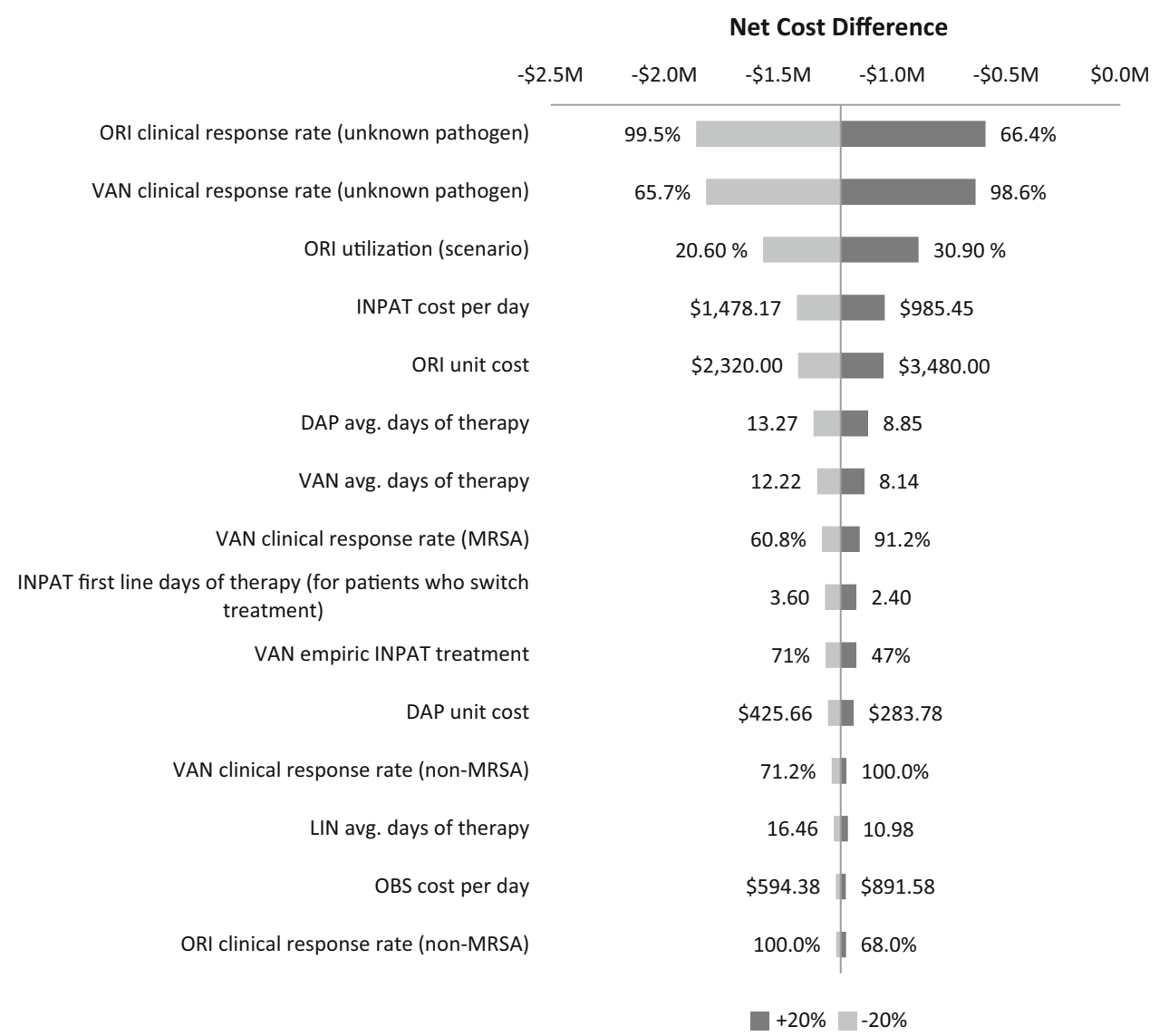

altogether, with treatment entirely in the ED or observation setting. While a portion of patients who do not respond to initial oritavancin therapy may ultimately be hospitalized (this was a clinical input for the model), the majority of patients receiving other intravenous antibacterials receive initial care in the hospital setting. The model results suggest that the higher acquisition price of oritavancin than of vancomycin is offset by reducing the total number of hospital admissions, decreasing drug administration costs, and reducing continued ambulatory infusions. Cost offsets were seen in both the inpatient and the outpatient setting through reduction in the need for repeated daily infusions and eliminating the need for an indwelling catheter, which also leads to fewer indwelling catheter complications. Though the base-case model assumes all patients receiving multi-day intravenous therapy received PICC lines, the sensitivity analysis indicates variations in PICC line utilization have a negligible $(<1 \%)$ impact on the hospital budget. Savings were also realized through the simplified dosing regimen (single intravenous dose of oritavancin) and lack of drug monitoring and other lab tests.

Though several economic models in ABSSSI have been previously published, few have incorporated both inpatient and outpatient costs as illustrated here [23, 30-32]. The strength of this model is therefore seen in the estimate of total hospital costs across all settings, including observation, admission, and discharge to outpatient care. Of note, no published health economic models were identified that incorporate the cost of observation care, which is becoming increasingly more common as hospitals strive to manage costs and reduce the burden of unnecessary hospital admissions. The model as described simulates a market mix of the most utilized empiric intravenous MRSA-active antibacterial agents and an expanded second-line treatment mix incorporating de-escalation, AE switching, and nonresponse. Many other models take a more narrow view comparing two agents or a more limited second-line armamentarium in specific patient scenario comparisons. Due to these differences, the model described here may be more representative of the total impact on the hospital budget, rather than only on patients whose treatment pathway mirrors the pathways selected in the other models. The results produced by this model are in the range of previously published cost estimates, lending additional credibility to the estimated results [23, 30-32].

Another strength of this model is the use of direct hospital cost estimates for most model inputs. The majority of previously published models have focused on payer costs as opposed to direct hospital costs, in part due to the difficulties in estimating and obtaining direct 
Table 4 Top 15 variables with the highest impact on net cost difference in univariate analysis

\begin{tabular}{|c|c|c|c|c|c|c|c|}
\hline \multirow[t]{2}{*}{ Input variable } & \multicolumn{4}{|c|}{ Net cost difference (US\$) } & \multirow{2}{*}{$\begin{array}{l}\text { Input } \\
\text { Min. }\end{array}$} & \multirow{2}{*}{$\begin{array}{l}\text { Input } \\
\text { Max. }\end{array}$} & \multirow{2}{*}{$\begin{array}{l}\text { Input } \\
\text { Base case }\end{array}$} \\
\hline & Minimum & Maximum & Range & $\begin{array}{l}\text { Explained } \\
\text { variation }(\%)^{\mathrm{a}}\end{array}$ & & & \\
\hline $\begin{array}{l}\text { ORI clinical response rate (unknown } \\
\text { pathogen) }\end{array}$ & $-591,840$ & $-1,861,825$ & $1,269,986$ & 40.76 & $66.4 \%$ & $99.5 \%$ & $82.9 \%$ \\
\hline $\begin{array}{l}\text { VAN clinical response rate (unknown } \\
\text { pathogen) }\end{array}$ & $-635,984$ & $-1,817,681$ & $1,181,697$ & 76.05 & $65.7 \%$ & $98.6 \%$ & $82.2 \%$ \\
\hline ORI utilization (scenario) & $-1,567,426$ & $-886,239$ & 681,186 & 87.77 & $20.60 \%$ & $30.90 \%$ & $25.75 \%$ \\
\hline INPAT cost per day & $-1,033,725$ & $-1,419,940$ & 386,215 & 91.54 & US\$985.45 & US\$1478.17 & US\$1231.81 \\
\hline ORI unit cost & $-1,039,294$ & $-1,414,371$ & 375,077 & 95.10 & US $\$ 2320.00$ & US $\$ 3480.00$ & US\$2900.00 \\
\hline DAP average days of therapy & $-1,107,094$ & $-1,346,571$ & 239,478 & 96.55 & 8.85 days & 13.27 days & 11.06 days \\
\hline VAN average days of therapy & $-1,123,285$ & $-1,330,380$ & 207,095 & 97.63 & 8.14 days & 12.22 days & 10.18 days \\
\hline VAN clinical response rate (MRSA) & $-1,144,245$ & $-1,309,420$ & 165,174 & 98.32 & $60.8 \%$ & $91.2 \%$ & $76.0 \%$ \\
\hline $\begin{array}{l}\text { INPAT first-line days of therapy (for } \\
\text { patients who switch treatment) }\end{array}$ & $-1,158,349$ & $-1,295,807$ & 137,458 & 98.80 & 2.40 days & 3.60 days & 3.00 days \\
\hline VAN empiric INPAT treatment & $-1,157,945$ & $-1,294,108$ & 136,163 & 99.27 & $47 \%$ & $71 \%$ & $59 \%$ \\
\hline DAP unit cost & $-1,170,498$ & $-1,283,167$ & 112,669 & 99.59 & US\$283.78 & US\$425.66 & US\$354.72 \\
\hline VAN clinical response rate (non-MRSA) & $-1,202,037$ & $-1,266,957$ & 64,920 & 99.69 & $71.2 \%$ & $100.0 \%$ & $89.0 \%$ \\
\hline LIN average days of therapy & $-1,195,803$ & $-1,257,862$ & 62,060 & 99.79 & 10.98 days & 16.46 days & 13.72 days \\
\hline OBS cost per day & $-1,204,582$ & $-1,249,082$ & 44,500 & 99.84 & US\$594.38 & US\$891.58 & US\$742.98 \\
\hline ORI clinical response rate (non-MRSA) & $-1,203,804$ & $-1,247,151$ & 43,347 & 99.89 & $68.0 \%$ & $100.0 \%$ & $85.0 \%$ \\
\hline
\end{tabular}

DAP daptomycin, INPAT inpatient, LIN linezolid, Max. maximum, Min. minimum, MRSA methicillin-resistant Staphylococcus aureus, OBS observation, $O R I$ oritavancin, VAN vancomycin

${ }^{\text {a }}$ Explained variation is cumulative

hospital costs. Often, Centers for Medicare \& Medicaid Services (CMS) reimbursement rates may be used as a proxy for costs when estimating hospital costs [27]. Here, published data on the actual direct costs of PICC lines (US\$413) were used, which were substantially lower than the CMS reimbursement rates (US\$850) [22, 28]. Using reimbursement rates in this instance would have overestimated the actual hospital costs associated with PICC line insertion. While reimbursement rates were used as proxy costs for some model variables, they were used sparingly in instances where applicable direct costs were not available. Sensitivity analyses indicate that these discrepancies were unlikely to have a substantial impact on the overall cost savings estimated by the model. Time and motion studies have been used in previous models to reflect hospital costs for inputs such as drug administration, though none have been recently conducted in the US [26, 29].

Several factors should be considered when interpreting the findings of this analysis. A number of simplifying assumptions were made in the development of the model that may impact model results; however, these are consistent with previously published decision-analytic models [23, 26, 31-33]. Direct costs from Gram-negative antibacterial coverage, additional concomitant medications (aside from those required to treat AEs), radiologic studies, and costs associated with use of isolation units were not included in the model as this was assumed to be consistent across all patients. Excluding these variables reduces the estimated total costs equally across patients, but would be unlikely to affect net budget impact. Some patients may also require therapy beyond second-line in order to achieve a successful clinical outcome. With the high response rates of currently available antibacterials (80-90\%), it is estimated that less than $4 \%$ of patients will require a third-line or later therapy. This figure is also in line with the reported 30-day readmission rate (3-4\%), which was demonstrated to have only a small impact on the overall budget (Fig. 2) $[34,35]$. Costs associated with monitoring tests for patients receiving oritavancin were not included as its prescribing information does not indicate that monitoring is necessary. However, sensitivity analysis reveals that even when costs associated with oritavancin are increased by US $\$ 580$, the treatment remains cost saving, indicating that with additional costs for monitoring the drug would remain cost saving. Finally, because of limited availability of data, it was assumed that outpatient drug-switching patterns were similar to those seen in the inpatient setting. This assumption was validated by clinical experts, and was considered unlikely to have a large impact on total budget impact; however, the exact magnitude and direction of this impact is unknown. 
The economic impact of antibacterial resistance was not considered for any drugs in this analysis. As is the case with other antibacterial agents, the efficacy of oritavancin may be impacted in the future by the development of resistance. However, clinical studies to date have not identified a high propensity for oritavancin resistance to emerge among indicated organisms, a finding that may be attributed to oritavancin's multiple mechanisms of action. Future emergence of resistance could change the economic results presented here. The impact of oritavancin on hospital-acquired infections (HAIs) is not included in this analysis and may require additional analysis. With more MRSA patients treated in the outpatient setting, it could be hypothesized that the rate of HAI may decline, thus further reducing costs. Additionally, with its single-dose administration, oritavancin may aid in improving quality measures such as reduced re-admissions from enhanced compliance, which may lower costs and have additional implications on reimbursement.

Other indirect costs and measures such as patient quality of life, treatment satisfaction, work productivity, and patient out-of-pocket costs were also excluded from this analysis. Use of single-dose oritavancin and thus avoidance of hospital admission and/or daily visits to the infusion center may enable patients to return to their normal activities of daily living sooner, potentially resulting in improved satisfaction, better quality of life, and reduced time and money costs of transport to and from an infusion center. Patients may also be able to return to work sooner, providing additional benefits to both patients and employers.

\section{Conclusions}

This budget impact analysis demonstrates that use of oritavancin in moderate to severe ABSSSI patients is expected to save hospitals substantial costs by reducing hospital admissions and decreasing costs associated with outpatient therapy. The positive economic impact holds regardless of whether the hospital has outpatient infusion services. Due to its single-dose administration, use of oritavancin may eliminate the need for daily intravenous drug administration and its associated costs. Use of oritavancin may also facilitate outpatient treatment of ABSSSI, thereby reducing the cost and resource use associated with hospital admissions.

\section{Compliance with Ethical Standards}

Funding This work was funded by The Medicines Company. ISJ, CW, and PLC are employees of ICON Health Economics, which provides consulting services to the pharmaceutical industry, including The Medicines Company.
WF and KAS are employees of The Medicines Company. SD and TPL provided consulting services to The Medicines Company.

DPN provided model input but did not receive an honorarium for contributions on this project. DPN is a Speaker for The Medicines Company.

This manuscript describes the development and results of an economic model, and does not describe research involving human participants and/or animals, thus no informed consent or Institutional Review Board approval was required.

While the manuscript was funded by The Medicines Company, the content and results reported in this manuscript were not subject to sponsor censorship and have been approved by all co-authors. This manuscript was developed in accordance with ICMJE (International Committee of Medical Journal Editors) guidelines and all authors have met the criteria for authorship as outlined in the Uniform Requirements for Manuscripts Submitted to Biomedical Journals. W. Fan and K.A. Sulham are employees of The Medicines Company and own stock or options. I.S. Jensen, P.L. Cyr and C. Wu were employees of ICON at the time of model and manuscript development, which provides consulting services to the pharmaceutical industry, including The Medicines Company, and may own stock or stock options. T.P. Lodise, D.P. Nicolau, and S. DuFour have provided funded consultancy services to ICON and The Medicines Company.

Open Access This article is distributed under the terms of the Creative Commons Attribution-NonCommercial 4.0 International License (http://creativecommons.org/licenses/by-nc/4.0/), which permits any noncommercial use, distribution, and reproduction in any medium, provided you give appropriate credit to the original author(s) and the source, provide a link to the Creative Commons license, and indicate if changes were made.

\section{References}

1. CDC. National Hospital Ambulatory Medical Care Survey: 2010 Emergency Department Summary Tables. http://www.cdc.gov/ nchs/data/ahcd/nhamcs_emergency/2010_ed_web_tables.pdf. Accessed 21 Sept 2014.

2. DiNubile MJ, Lipsky BA. Complicated infections of skin and skin structures: when the infection is more than deep. J Antimicrob Chemother. 2004;53 Suppl 2:ii37-50.

3. LaPensee K, Fan W. Economic burden of hospitalization with antibiotic treatment for ABSSSI in the US: an analysis of the Premier hospital database [poster]. In: ISPOR 17th annual international meeting, Washington, DC; 2-6 June 2012.

4. Tice A. Oritavancin: a new opportunity for outpatient therapy of serious infections. Clin Infect Dis. 2012;54:S239-43.

5. Ki V, Rotstein C. Bacterial skin and soft tissue infections in adults: a review of their epidemiology, pathogenesis, diagnosis, treatment and site of care. Can J Infect Dis Med Microbiol. 2008;19(2):173-84.

6. Moran GM, Krishnadasan A, Gorwitz RJ, Fosheim GE, McDougal LK, Carey RB, EMERGEncy ID Net Study Group, et al. Methicillin-resistant $S$. aureus infections among patients in the emergency department. N Engl J Med. 2006;355:666-74.

7. Stevens DL, Bisno AL, Chambers HF, Dellinger EP, Goldstein EJ, Gorbach SL, Infectious Diseases Society of America, et al. Practice guidelines for the diagnosis and management of skin and soft tissue infections: 2014 update by the Infectious Diseases Society of America. Clin Infect Dis. 2014;2014(59):e10-52. 
8. Liu C, Bayer A, Cosgrove SE, Daum RS, Fridkin SK, Gorwitz $\mathrm{RJ}$, et al. Clinical practice guidelines by the Infectious Disease Society of America for the treatment of methicillin-resistant Staphylococcus aureus infections in adults and children: executive summary. Clin Infect Dis. 2011;52(3):285-92.

9. Vancomycin Injection, USP in GALAXY Plastic Container (PL 2040) for intravenous use only. http://www.accessdata.fda.gov/ drugsatfda_docs/label/2009/050671s014lbl.pdf. Accessed 9 Apr 2015.

10. CUBICIN (daptomycin for injection) for intravenous use. Cubist Pharmaceuticals; 2013. http://cubicin.com/pdf/PrescribingInformation.pdf. Accessed 14 Oct 2014

11. Teflaro (ceftaroline fosamil) for injection [prescribing information]. Actavis; 2015. http://pi.actavis.com/data_stream.asp? product_group $=1915 \& \mathrm{p}=$ pi\&language=E. Accessed 9 Apr 2015.

12. ZYVOX (linezolid) injection, tablets and oral suspension [prescribing information]. Pfizer; 2014. http://labeling.pfizer.com/ showlabeling. aspx?id=649. Accessed 14 Oct 2014 .

13. ORBACTIV (oritavancin) for injection, for intravenous use. The Medicines Company; 2014 [prescribing information]. http:// www.orbactiv.com/pdfs/orbactiv-prescribing-information.pdf. Accessed 29 Oct 2014.

14. Sulham K, LaPensee K, Fan W, Lodise TP. Severity and costs of acute bacterial skin and skin structure infections by treatment setting: an application of the Eron classification to a real-world database [poster]. In: ISPOR 19th annual international meeting, Montreal; 31 May-4 June 2014.

15. Corey GR, Kabler H, Mehra P, Mehra P, Gupta S, Overcash JS, SOLO I Investigators, et al. Single-dose oritavancin in the treatment of acute bacterial skin infections. N Engl J Med. 2014;370:2180-90.

16. Corey GR, Good S, Jiang H, Moeck G, Wikler M, Green S, SOLO II Investigators, et al. Single-dose oritavancin versus 7-10 days of vancomycin in the treatment of gram-positive acute bacterial skin and skin structure infections: the SOLO II noninferiority study. Clin Infect Dis. 2015;60(2):254-62.

17. Thom H, Thompson J, Scott D, Halfpenny N, Sulham K, Corey GR. Comparative efficacy of antibiotics for the treatment of acute bacterial skin and skin structure infections (ABSSSI): a systematic review and network meta-analysis. Curr Med Res Opin. 2015;31(8):1539-51.

18. Fan W, Mao J, Iorga S, Zarotsky V, LaPensee KT, Becker L. Care pathway and health care cost for acute bacterial skin and skin structure infection subjects with antibiotic treatment in the US: analysis of a real world database [poster]. In: ISPOR 18th Annual International Meeting, New Orleans; 18-22 May 2013.

19. Premier Research Services. Premier Research Database 2012. Charlotte, NC.

20. Schrock JW, Laskey S, Cydulka RK. Predicting observation unit treatment failures in patients with skin and soft tissue infections. Int J Emerg Med. 2008;1(2):85-90.

21. Hostetler B, Leikin JB, Timmons JA, Hanashiro PK, Kissane K. Patterns of use of an emergency department-based observation unit. Am J Ther. 2002;9(6):499-502.

22. Robinson MK, Mogensen KM, Grudinskas GF, Kohler S, Jacobs DO. Improved care and reduced costs for patients requiring peripherally inserted central catheters: the role of bedside ultrasound and a dedicated team. J Parenter Enteral Nutr. 2005;29(5):374-9.

23. Stephens JM, Gao X, Patel DA, Verheggen BG, Shelbaya A, Haider S. Economic burden of inpatient and outpatient antibiotic treatment for methicillin-resistant Staphylococcus aureus complicated skin and soft-tissue infections: a comparison of linezolid, vancomycin, and daptomycin. Clinicoecon Outcomes Res. 2013;5:447-57.
24. Medi-Span Price Rx ${ }^{\circledR}$. https://pricerx.medispan.com/. Accessed 17 July 2014.

25. Bureau of Labor Statistics. CPI detailed report data, July 2014. http://www.bls.gov/cpi/cpid1407.pdf. Accessed 29 Oct 2014.

26. Huang X, Beresford E, Lodise T, Friedland HD. Ceftaroline fosamil use in hospitalized patients with acute bacterial skin and skin structure infections: budget impact analysis from a hospital perspective. Am J Health Syst Pharm. 2013;70:1057-64.

27. MedPAC. June 2014: a data book: health care spending and the Medicare program. http://www.medpac.gov/documents/publica tions/jun14databookentirereport.pdf. Accessed 13 Nov 2014.

28. AMA code manager. https://ocm.ama-assn.org/OCM/mainMenu. do. Accessed 20 May 2014.

29. van Zanten AR, Engelfriet PM, van Dillen K, van Veen M, Nuijten MJ, Polderman KH. Importance of nondrug costs of intravenous antibiotic therapy. Crit Care. 2003;7(6):R184-90.

30. Barron J, Turner R, Jaeger M, Adamson W, Singer J. Comparing the use of intravenous antibiotics under the medical benefit with the use of oral antibiotics under the pharmacy benefit in treating skin and soft tissue infections. Manag Care. 2012;21(9):44-52.

31. Bounthavong M, Zargarzadeh A, Hsu DI, Vanness DJ. Cost-effectiveness analysis of linezolid, daptomycin, and vancomycin in methicillin-resistant Staphylococcus aureus: complicated skin and skin structure infection using Bayesian methods for evidence synthesis. Value Health. 2011;14(5):631-9.

32. Bounthavong M, Hsu DI, Okamoto MP. Cost-effectiveness analysis of linezolid vs. vancomycin in treating methicillin-resistant Staphylococcus aureus complicated skin and soft tissue infections using a decision analytic model. Int J Clin Pract. 2009;63(3):376-86.

33. De Cock E, Sorensen S, Levrat F, Besnier JM, Dupon M, Guery $\mathrm{B}$, et al. Cost-effectiveness of linezolid versus vancomycin for hospitalized patients with complicated skin and soft-tissue infections in France. Med Mal Infect. 2009;39(5):330-40.

34. Nguyen HH. Hospitalist to home: outpatient parenteral antimicrobial therapy at an academic center. Clin Infect Dis. 2010;51(Suppl 2):S220-3.

35. Jauregui LE, Babazadeh S, Seltzer E, Goldberg L, Krievins D, Frederick M, et al. Randomized, double-blind comparison of once-weekly dalbavancin versus twice-daily linezolid therapy for the treatment of complicated skin and skin structure infections. Clin Infect Dis. 2005;41(10):1407-15.

36. Stryjewski ME, Graham DR, Wilson SE, O'Riordan W, Young D, Lentnek A, Assessment of Telavancin in Complicated Skin and Skin-Structure Infections Study, et al. Telavancin versus vancomycin for the treatment of complicated skin and skinstructure infections caused by gram-positive organisms. Clin Infect Dis. 2008;46(11):1683-93.

37. Stevens DL, Smith LG, Bruss JB, McConnell-Martin MA, Duvall SE, Todd WM, et al. Randomized comparison of linezolid (PNU100766) versus oxacillin-dicloxacillin for treatment of complicated skin and soft tissue infections. Antimicrob Agents Chemother. 2000;44(12):3408-13.

38. Arbeit RD, Maki D, Tally FP, Campanaro E, Eisenstein BI, Daptomycin 98-01 and 99-01 Investigators. The safety and efficacy of daptomycin for the treatment of complicated skin and skin-structure infections. Clin Infect Dis. 2004;38(12):1673-81.

39. Dunbar LM, Milata J, McClure T, Wasilewski MM, SIMPLIFI Study Team. Comparison of the efficacy and safety of oritavancin frontloaded dosing regimens to daily dosing: an analysis of the SIMPLIFI trial. Antimicrob Agents Chemother. 2011;55(7):3476-84.

40. PDR Network, LLC. http://www.pdrnetwork.com/. Accessed 15 Dec 2013. 